PharmacoEconomics \& Outcomes News 870, p27 - 23 Jan 2021

\title{
SARS-CoV-2 testing with saliva versus nasopharyngeal swabs: similar sensitivity, lower cost
}

Testing for severe acute respiratory syndrome coronarvirus 2 (SARS-CoV-2) infection using saliva samples has similar sensitivity than using nasopharyngeal swabs and is less costly, according to the results of a literature review published in Annals of Internal Medicine.

The review was conducted by researchers from Canada to compare the sensitivity for SARS-CoV-2 detection between saliva and nasopharyngeal swabs and to estimate the incremental cost per additional SARS-CoV-2 infection detected with nasopharyngeal swabs.

The search of four databases from January to November 2020 revealed 37 studies that reported accuracy of salivabased sampling compared with nasopharyngeal swabs for SARS-CoV-2 detection. The analysis of data from a total of 7332 paired saliva samples and nasopharyngeal swabs showed that the sensitivity of saliva was 3.4 percentage points lower than that of nasopharyngeal swabs. Nevertheless, the analyses stratified by population characteristics showed no statistically significant difference in sensitivity between these specimens for SARS-CoV-2 detection. The economic evaluation of the subgroup of undiagnosed persons presenting for SARS-CoV-2 testing showed 79 more persons in a cohort of 100000 persons would be detected using nasopharyngeal swabs versus saliva, assuming a SARS-CoV-2 prevalence of $1 \%$, at an incremental cost of $\$$ US 8093 per additional SARS-CoV-2 infection detected. The researchers note that saliva sampling has the advantages of less invasiveness, reduced need for trained healthcare professionals and lower risk for occupational exposure. "Saliva sampling should replace nasopharyngeal swabs in most populations being tested for SARS-CoV-2," suggest the researchers.

Bastos ML, et al. The Sensitivity and Costs of Testing for SARS-CoV-2 Infection With Saliva Versus Nasopharyngeal Swabs : A Systematic Review and Meta-analysis. Annals of Internal Medicine : 12 Jan 2021. Available from: URL: http://doi.org/10.7326/M20-6569 